\title{
Perfil sociodemográfico e clínico de usuários de substâncias psicoativas atendidos em hospital filantrópico acreditado*
}

\author{
Jaqueline Fátima de Souza ${ }^{1}$ \\ (D) https://orcid.org/0000-0002-9619-3091 \\ Marcos Hirata Soares ${ }^{1}$ \\ (D) https://orcid.org/0000-0002-1391-9978 \\ Jessica Andrade Tiziani ${ }^{1}$ \\ (ID) https://orcid.org/0000-0003-0277-3616
}

\footnotetext{
A publicação deste artigo na Série Temática "Recursos Humanos em Saúde e Enfermagem" se insere na atividade 2.2 do Termo de Referência 2 do Plano de Trabalho do Centro Colaborador da OPAS/OMS para o Desenvolvimento da Pesquisa em Enfermagem, Brasil. Artigo extraído da dissertação de mestrado "Atendimento ao paciente com abuso de substâncias psicoativas em um hospital geral", apresentada à Universidade Estadual de Londrina, Londrina, PR, Brasil.

${ }^{1}$ Universidade Estadual de Londrina, Pós Graduação, Londrina, PR, Brasil.
}

Objetivo: descrever o perfil de usuários de substâncias psicoativas, seu desfecho e complicações clínicas. Método: estudo transversal com 67 pacientes em um hospital geral filantrópico acreditado. Análise descritiva exploratória a partir dos dados obtidos com os instrumentos Addicttion Severity Index - 6 e Alcohol Smoking and Substance Involvement Screening Test. Resultados: houve predominância do sexo masculino em $83,5 \%$ das causas de admissão, $32,8 \%$ estavam relacionadas ao sistema musculoesquelético, e $43,3 \%$ dos pacientes desconheciam comorbidades prévias. Prevalência de 73,7\% do uso abusivo de álcool, 44,8\% tiveram pontuação superior a 27 pontos, indicando necessidade de encaminhamento para especialidade psiquiátrica. Conclusão: carecem esforços no sentido de aprimorar e amadurecer processos para garantir a qualidade do serviço e segurança do paciente em envolvimento com substâncias psicoativas em hospital geral filantrópico.

Descritores: Alcoolismo; Drogas Ilícitas; Hospital Geral; Saúde Mental.

\section{Como citar este artigo}

Souza JF, Soares MH, Tiziani JA. Sociodemographic and clinical profile of users of psychoactive substances in an accredited philanthropic hospital. SMAD, Rev Eletrônica Saúde Mental Álcool Drog. 2021 jul.-set.;17(3):7-17. doi: https://dx.doi.org/10.11606/issn.1806-6976.smad.2021.163560 


\section{Sociodemographic and clinical profile of users of psychoactive substances in an accredited philanthropic hospital}

Objective: to describe the profile of users of psychoactive substances, their outcomes and clinical complications. Method: a cross-sectional study with 67 patients in an accredited philanthropic general hospital. Exploratory descriptive analysis based on data obtained with the addiction severity index -6 and the Alcohol Smoking Substance and Involvement Screening Test. Results: there was predominance of males in $83.5 \%$ of the causes of admission, $32.8 \%$ were related to the musculoskeletal system, and $43.3 \%$ of the patients were unaware of previous comorbidities. Prevalence of $73.7 \%$ of alcohol abuse, $44.8 \%$ scored higher than 27 points, indicating the need for referral to the psychiatric specialty. Conclusion: efforts are needed to improve and mature processes to ensure the quality of service and patient safety in involvement with psychoactive substances in a philanthropic general hospital.

Descriptors: Alcoholism; Illicit Drugs; General Hospital; Mental Health.

\section{Perfil sociodemográfico y clínico de usuarios de sustancias psicoactivas en un hospital filantrópico acreditado}

Objetivo: este estudio presenta el perfil de los usuarios de sustancias psicoactivas, el resultado, las complicaciones clínicas y el contexto familiar. Método: estudio transversal en un hospital general filantrópico acreditado con 67 pacientes. Un análisis exploratorio descriptivo basado en los datos obtenidos con los instrumentos Addicttion Severity Index - 6 y Alcohol Smoking and Substance Involvement Screening Test. Resultados: hubo un predominio del sexo masculino en el 83,5\% de las causas de admisión, el 32.8\% fueron relacionados con el sistema muscular esquelético, y el $43,3 \%$ de los pacientes desconocían las comorbilidades anteriores. Prevalencia del $73,7 \%$ del abuso de alcohol, el $44,8 \%$ obtuvo más de 27 puntos, lo que indica la necesidad de derivación a la especialidad psiquiátrica. Conclusión: el contexto familiar es también un factor que contribuye a la vulnerabilidad del individuo. Por lo tanto, se advierte la necesidad de realizar esfuerzos para mejorar y madurar los procesos para garantizar la calidad del servicio y la seguridad del paciente en el contexto de la atención de salud mental en un hospital general.

Descriptores: Alcoholismo; Drogas Ilícitas; Hospital General; Salud Mental. 


\section{Introdução}

Historicamente, a reforma psiquiátrica foi marcada por implementações de serviços adeptos ao modelo de atenção psicossocial, em que os Centros de Atenção Psicossocial (CAPS) passaram a exercer um papel fundamental e centrado no cuidado em saúde mental(1). Contudo, vários desafios são enfrentados no contexto da reforma psiquiátrica, principalmente a integração de serviços não especializados em saúde mental. O senso comum ainda entende que o sofrimento psíquico, de certa forma, é uma irracionalidade, o que sugere que o modelo manicomial seria o mais apropriado(2), contrariando o pressuposto no Sistema Único de Saúde (SUS) ${ }^{(3)}$.

Os hospitais gerais de média e alta complexidade iniciaram, então, a integração da Rede de Atenção Psicossocial (RAPS), por meio da Portaria no 3.088/2011, propondo atendimento de assistência integral e possibilitando atuação de profissionais com formação específica como regem os princípios e diretrizes do SUS ${ }^{(4-5)}$.

Serviços hospitalares recebem pacientes com risco de morte pelo abuso de álcool e drogas incessantemente. O abuso do álcool, especificamente, só foi reconhecido pela Organização Mundial da Saúde (OMS) como uma doença mental a partir de 1977. Desde então, a OMS considera que de 10 a $12 \%$ da população mundial apresenta problemas de abuso do álcool, associado também a fatores socioeconômicos(4).

De acordo com relatório de World Drug Report, houve aumento de $39 \%$ do consumo de drogas entre os anos de 2001 e 2015, elevando em 60\% as causas de mortes no mundo, tendo como principal característica a prevalência de consumo maior entre os homens. No Brasil, cerca de $3 \%$ da população geral sofre de transtornos mentais, sendo que, em $6 \%$ dessa população, o transtorno apresentado relaciona-se ao abuso de álcool e outras drogas, dos quais $12 \%$ necessitam de atendimento contínuo relacionado aos transtornos mentais(5).

As evidências encontradas atualmente na população brasileira apontam que da população total com transtornos mentais, $6 \%$ a $8 \%$ estão relacionados ao uso abusivo de álcool(6). Embora haja vasta literatura abordando a caracterização desta população, há escassez de estudos em hospitais filantrópicos acreditados que têm como objetivo a segurança do paciente com base em metas e indicadores de avaliação do atendimento multiprofissional, compreendendo as avaliações especializadas para saúde mental e o seu desfecho clínico quando necessário.

Dessa forma, teve-se como objetivo descrever o perfil socioeconômico dos usuários de álcool e outras drogas internados em um hospital geral acreditado, assim como o desfecho de suas complicações clínicas após sua internação.

\section{Método}

Trata-se de um estudo transversal, realizado em um hospital geral filantrópico acreditado pela Organização Nacional de Acreditação (ONA), na região sul do Brasil. Esse hospital tem 338 leitos, mas sem leitos psiquiátricos. A pesquisa ocorreu entre novembro de 2018 e abril de 2019. A amostra foi não probabilística por conveniência, sendo assim intencional ocorrendo a seleção do serviço em instituição de formação de alunos.

Para inclusão na amostra foram selecionados pacientes acima de 18 anos, cuja internação fosse superior a 24 horas, com histórico de uso de álcool ou outras drogas, de ambos os sexos e com qualquer doença ou estado clínico, havendo associação preestabelecida com abuso de substâncias psicoativas. Foram excluídos pacientes com diagnóstico de transtorno mental severo, indivíduos com deterioração física, pobreza de linguagem e/ou intoxicação que limitasse a aplicação do instrumento norteador. Dentre esses, a partir da consulta ao prontuário eletrônico, fez-se a exclusão de 22 pacientes, dos quais nove não apresentaram melhoras das condições clínicas e nove evoluíram a óbito antes da primeira avaliação. Após o convite verbal da pesquisadora, dois não aceitaram participar e outros dois solicitaram alta antes do convite verbal. Realizou-se busca ativa nas unidades de internação e unidades de terapia intensiva. Ao abordar cada sujeito, explicou-se o objetivo e condições da pesquisa, que envolvia a aplicação do Termo de Consentimento Livre e Esclarecido (TCLE) e, sequencialmente, o Alcohol, Smoking and Substance Involvement Screening Test (ASSIST) e o Addicttion Severity Index (ASI6).

Para a coleta de dados, utilizou-se o teste ASSIST que foi validado no Brasil por Henrique et al. (2004)(7), o qual tem como objetivo a identificação de problemas relacionados ao uso de álcool e substâncias psicoativas, por meio de pontuação total entre zero e $>27$ pontos. A confiabilidade foi feita por meio de teste-reteste pelos coeficientes Kappa, que se deu entre 0,58 e 0,90.

A escala ASI6 traduzida e validada por Felix Henrique Paim Kessler em 2005 foi delineada para obter informações relacionadas aos aspectos socioeconômicodemográficos e culturais associados ao abuso de álcool e outras drogas. Essa escala contém oito eixos para compreensão psicossocial do indivíduo. Para este estudo foram utilizadas as seguintes abordagens: gênero, faixa etária, raça, estado civil, renda, fonte de renda, escolaridade, dependentes da renda e carteira assinada. A escala ASI6 teve a confiabilidade por meio de testereteste; para as subescalas utilizou-se alfa de Cronbach, que variou de 0,64 a 0,95(8).

Além da aplicação dos instrumentos, foram coletados dados do prontuário eletrônico, com informações do período de internação e assistência prestada pelos profissionais: psicólogos, psiquiatras, assistentes sociais, entre outras especialidades médicas específicas para o 
tratamento clínico. Para a análise dos dados, optou-se pela análise estatística exploratória, utilizando medidas de tendência central para a apresentação das variáveis. Para todo o processo de pesquisa, a pesquisadora recebeu treinamento de 40 horas, para realização da Intervenção Breve e aplicação dos instrumentos de coleta de dados, durante sua participação no grupo de pesquisa.

\section{Resultados}

Houve predominância de internações do sexo masculino no hospital geral, sendo de $83,5 \%$ em comparação às internações do sexo feminino, que foram de $16,4 \%$ (Tabela 1). De acordo com a idade, 37,3\% estão na faixa etária entre 31 e 50 anos, precedidos de $28,4 \%$ com faixa etária entre 51 e 70 anos. Quanto ao estado civil, tem-se $53,7 \%$ de solteiros, seguido de $25,4 \%$ de casados ou amasiados. Para a variável etnia, $80,6 \%$ se auto declararam como brancos, $16,4 \%$ como pardos e mestiços e $3 \%$ como negros.

Tabela 1 - Caracterização e perfil dos pacientes em abuso de álcool e outras drogas em um hospital geral. Londrina, PR, Brasil, 2019

\begin{tabular}{lcc}
\hline Variável & Frequência & $\%$ \\
\hline Gênero & 56 & \\
Masculino & 11 & 83,5 \\
Feminino & 67 & 16,4 \\
Total & & 100,0 \\
\hline
\end{tabular}

\begin{tabular}{lcc}
\hline Faixa etária & & \\
18 a 30 anos & 15 & 22,4 \\
31 a 50 anos & 25 & 37,3 \\
51 a 70 anos & 19 & 28,4 \\
71 a 90 anos & 8 & 11,9 \\
Total & 67 & 100,0 \\
\hline Etnia & 2 & 3,0 \\
Negra & 54 & 80,6 \\
Branca & 11 & 16,4 \\
Parda/mestiça & 67 & 100,0 \\
\hline Total &
\end{tabular}

\section{Estado civil}

\begin{tabular}{lcc} 
Casado ou com parceiro & 17 & 25,4 \\
$\begin{array}{l}\text { Com parceiro } \\
\text { anteriormente }\end{array}$ & 14 & 20,9 \\
Solteiro & 36 & 53,7 \\
Total & 67 & 100,0 \\
\hline
\end{tabular}

Quanto à renda per capita (Tabela 2), 53,7\% desses indivíduos sobrevivem com uma renda de até dois salários mínimos, 17,9\% não possuem renda mensal, 25,4\% têm sua fonte por meio de trabalhos informais e $62,9 \%$ não tinham carteira de trabalho assinada. A pesquisa também demonstrou um índice de baixa escolaridade, em que $52,2 \%$ possuem apenas o ensino fundamental; fator que pode ter influenciado e dificultado a inserção no mercado de trabalho, pois $62,9 \%$ dos indivíduos tiveram seu último trabalho sem carteira assinada. Quanto às condições de moradia, 26,3\% relataram já ter habitado em abrigo supervisionado.

Tabela 2 - Distribuição da renda per capita dos indivíduos e familiares e sua escolaridade. Londrina, PR, Brasil, 2019

\begin{tabular}{|c|c|c|}
\hline Variável & Frequência & $\%$ \\
\hline \multicolumn{3}{|l|}{ Renda } \\
\hline Sem renda & 12 & 17,9 \\
\hline$<1$ salário & 24 & 35,8 \\
\hline 1-2 salários & 20 & 29,9 \\
\hline 2-5 salários & 4 & 6,0 \\
\hline$>5$ salários & 7 & 10,4 \\
\hline Total & 67 & 100,0 \\
\hline \multicolumn{3}{|l|}{ Fonte de renda } \\
\hline Emprego & 8 & 11,9 \\
\hline $\begin{array}{l}\text { Aposentadoria ou } \\
\text { invalidez }\end{array}$ & 15 & 22,4 \\
\hline Famílias e outros & 16 & 23,9 \\
\hline Dinheiro ilegal & 4 & 6,0 \\
\hline Trabalho não formal & 17 & 25,4 \\
\hline Nenhuma fonte de renda & 7 & 10,4 \\
\hline Total & 67 & 100,0 \\
\hline \multicolumn{3}{|l|}{ Dependentes da renda } \\
\hline Não & 49 & 73,1 \\
\hline Sim & 18 & 26,9 \\
\hline Total & 67 & 100,0 \\
\hline \multicolumn{3}{|l|}{ Escolaridade } \\
\hline Não alfabetizado & 3 & 4,5 \\
\hline Fundamental & 35 & 52,2 \\
\hline Ens. médio & 27 & 40,3 \\
\hline Ens. superior & 2 & 3,0 \\
\hline Total & 67 & 100,0 \\
\hline
\end{tabular}

Quanto aos aspectos clínicos, foi identificado que $43,3 \%$ dos pacientes internados não tinham comorbidades ou diagnóstico estabelecido previamente. Dos indivíduos que possuíam conhecimento de comorbidades, havia 23,9\% com doenças do sistema gastrointestinal, e desses, $22,9 \%$ tinham cirrose hepática alcoólica. Em relação às doenças do sistema cardiovascular, 28,4\% desenvolveram hipertensão arterial. No entanto, 32,8\% foram internados por consequência de múltiplas fraturas. 
Dentre os demais achados neste estudo, foram admitidos $17,9 \%$ dos pacientes por consequências de alterações do sistema gastrointestinal, onde a maior incidência foi de doença hepática, e com o mesmo percentual foram admitidos pacientes com alterações neurológicas (trauma cranioencefálico e acidente vascular cerebral).

O tempo médio de permanência (TMP) foi de um a sete dias para $53,7 \%$ dos pacientes, e maior que 15 dias para $7,5 \%$ deles (Tabela 3 ). Considerando que $13,4 \%$ desses internados necessitaram de Unidade de Terapia Intensiva (UTI) e tiveram um tempo médio de permanência de seis a sete dias; ao serem admitidos na UTI, apresentaram o Simplified Acute Physiology Score 3 (SAPS 3- validação e refinamento de índices prognósticos em pacientes graves admitidos em UTI) preenchido. Assim, 9,2\% desses pacientes tiveram a pontuação menor que $20 \%$, e 9,2\% a pontuação entre 21 e $40 \%$. O grau de dependência de todos os indivíduos, conforme a escala de Fugulin (classificação de pacientes, de acordo com o grau de dependência), 34,2\% foram classificados com grau de dependência III, 30,1\% com grau de dependência II e $27,1 \%$ com grau de dependência IV.

Tabela 3 - Frequência e porcentagem das doenças físicas, comorbidades e o tempo de internação em hospital geral e em unidade de terapia intensiva. Londrina, PR, Brasil, 2019

\begin{tabular}{|c|c|c|}
\hline Variável & Frequência & $\%$ \\
\hline \multicolumn{3}{|l|}{ Causas de admissão } \\
\hline Sistema musculoesquelético & 22 & 32,8 \\
\hline Sistema gastrointestinal & 12 & 17,9 \\
\hline Sistema neurológico & 10 & 14,9 \\
\hline Outros & 23 & 34,3 \\
\hline Total & 67 & 100,0 \\
\hline \multicolumn{3}{|l|}{ Comorbidades } \\
\hline Nenhuma & 29 & 43,3 \\
\hline Sistema cardiovascular & 19 & 28,4 \\
\hline Sistema gastrointestinal & 16 & 23,9 \\
\hline Sistema respiratório & 1 & 1,5 \\
\hline Outras & 2 & 3,0 \\
\hline Total & 67 & 100,0 \\
\hline \multicolumn{3}{|l|}{ Tempo médio em hosp. geral } \\
\hline 1 a 7 dias & 36 & 53,7 \\
\hline 8 a 14 dias & 26 & 38,8 \\
\hline$>15$ dias & 5 & 7,5 \\
\hline Total & 67 & 100,0 \\
\hline
\end{tabular}

Correlacionando o percentual de grau de dependência com a complicação do sistema do corpo humano, observou-se que os indivíduos internados por complicações do sistema gastrointestinal demandaram maior tempo de atendimento e atenção da equipe de enfermagem quando comparado com as demais complicações de outros sistemas do corpo humano. Desses, 13,4\% foram classificados como grau de dependência IV em algum momento do período da internação. Já os atendidos por complicações do sistema musculoesquelético tiveram uma média percentual de 14,9\%, com grau de dependência III.

Foi possível constatar que $73,7 \%$ dos sujeitos faziam uso de bebida alcoólica, 38,8\% tiveram uma pontuação entre 11-26 conforme teste ASSIST, pontuação que indica necessidade de intervenção breve; ainda, 44,8\% destes tiveram uma pontuação acima de 27 e necessitavam ser encaminhados para tratamento específico. Dos pesquisados, 39,9\% também faziam uso de tabaco, sendo que $19,4 \%$ dos indivíduos tiveram pontuação maior que 27. Quando avaliado o uso de substâncias ilícitas, $25,4 \%$ dos sujeitos foram classificados como prováveis dependentes de maconha, 28,4\% com uso de cocaína/ crack, e $72,6 \%$ fizeram o primeiro uso de droga ilícita quando tinham entre 10 e 17 anos de idade, 27,6\% após os 18 anos (Tabela 4).

Considerando o atendimento multiprofissional, $38,8 \%$ receberam atendimento de um assistente social, $9 \%$ receberam atendimento de psicólogo e 3\% tiveram interconsulta de um médico psiquiatra, o que resulta em $55,2 \%$ dos pacientes em uso de substância psicoativa sem receber atendimento na esfera psicossocial.

Quanto à variável "uso de substância psicoativa", um indivíduo poderia fazer uso de mais de uma substância psicoativa, não possibilitando, assim, um percentual de $100 \%$.

Tabela 4 - Percentual do uso de substâncias psicoativas e desfecho do período de internação. Londrina, PR, Brasil, 2019

\begin{tabular}{lcc}
\hline Variável & Frequência & $\%$ \\
\hline \% do uso de substância psicoativa & & \\
Álcool & 56 & 73,7 \\
Tabaco & 30 & 39,5 \\
Drogas ilícitas & 29 & 38,2 \\
\hline Álcool & & \\
Não faz uso & 7 & 10,4 \\
0-10 pontos & 4 & 6,0 \\
11-26 pontos & 26 & 38,8 \\
$>$ 27 pontos & 30 & 44,8 \\
Total & 67 & 100,0 \\
\hline Tabaco & & \\
Não faz uso & 34 & 50,7 \\
\hline
\end{tabular}




\begin{tabular}{lcc}
\hline Variável & Frequência & $\%$ \\
\hline 0-3 pontos & 2 & 3,0 \\
4-26 pontos & 18 & 26,9 \\
$>$ 27 pontos & 13 & 19,4 \\
Total & 67 & 100,0 \\
\hline
\end{tabular}

em algum momento da vida, sendo $100 \%$ desses do sexo feminino.

Tabela 5 - Identificação do convívio social do indivíduo perante o consumo de substância psicoativa. Londrina, PR, Brasil, 2019

\begin{tabular}{lcc}
\hline Maconha & & \\
Não faz uso & 50 & 74,6 \\
0-3 pontos & 4 & 6,0 \\
11-26 pontos & 4 & 6,0 \\
$>$ 27 pontos & 9 & 13,4 \\
Total & 67 & 100,0 \\
\hline Cocaína/crack & & \\
Não faz uso & 48 & 71,6 \\
0-3 pontos & 2 & 3,0 \\
$4-26$ pontos & 4 & 6,0 \\
$>$ 27 pontos & 13 & 19,4 \\
Total & 67 & 100,0 \\
\hline Desfecho clínico & & \\
Óbito & 9 & 13,4 \\
Alta médica & 50 & 74,6 \\
Alta a pedido & 7 & 10,4 \\
Evasão & 1 & 1,5 \\
Total & 67 & 100,0 \\
\hline
\end{tabular}

É possível que a adesão ao tratamento tenha sido influenciada quando o indivíduo retornou ao seu convívio social, em meio a hábitos costumeiros do consumo de SPA, assim como pode ter tido influxo quanto a sua satisfação com o relacionamento e o estado de harmonia na convivência com familiares e amigos. Segundo a Tabela 5, identificou-se que 52,2 \% dos indivíduos têm em seu convívio pessoas que fazem uso de SPA. Dentre esses, 40,3\% são familiares e $11,9 \%$ são amigos, que provavelmente fazem uso de SPA. Dentre esses, $52,2 \%$ relataram não estarem nada satisfeitos com o seu relacionamento com as pessoas de seu convívio, $16,4 \%$ disseram estar moderadamente satisfeitos e apenas $10,4 \%$ relataram ter um convívio social familiar extremamente satisfeito. Além disso, 37,3\% dos indivíduos afirmam não poder contar com pessoas nos momentos difíceis.

O relacionamento familiar e convívio social resultaram em agressão física para $44,8 \%$ dos indivíduos. Para 71,6\% desses, a primeira agressão sofrida se deu com menos de 10 anos de vida, 19,7\% sofreu a agressão entre os 11 e 20 anos de idade, e em apenas 5,3\% a agressão ocorreu acima dos 20 anos de idade. Dos indivíduos que sofreram agressão física, 5,3\% também sofreram agressão sexual

\begin{tabular}{lcc}
\hline Variável & Frequência & $\%$ \\
\hline Com quem mora atualmente? & \\
Sozinho & 16 & 23,9 \\
Cônjuge/parceiro & 20 & 29,9 \\
Filhos & 2 & 3,0 \\
Pais & 16 & 23,9 \\
Outros adultos não & 13 & 19,4 \\
parentes & 67 & 100,0 \\
Total & 32 & 47,8 \\
\hline Quais pessoas do convívio fazem uso de SPA? & \\
Ninguém & & 11,9 \\
Parceiro ou & 27 & 100,0 \\
parentes & 67 & \\
Amigo & & \\
Total & & \\
\hline
\end{tabular}

Qual sua satisfação com o relacionamento interpessoal com família e amigos?

\begin{tabular}{lcc} 
Nada satisfeito & 35 & 52,2 \\
Levemente satisfeito & 4 & 6,0 \\
$\begin{array}{l}\text { Moderadamente } \\
\text { satisfeito }\end{array}$ & 10 & 14,9 \\
$\begin{array}{l}\text { Consideravelmente } \\
\text { satisfeito }\end{array}$ & 11 & 16,4 \\
$\begin{array}{l}\text { Extremamente } \\
\text { satisfeito }\end{array}$ & 7 & 10,4 \\
Total & 67 & 100,0 \\
\hline
\end{tabular}

É importante para você ter um relacionamento com outras pessoas?

\begin{tabular}{ccc} 
Não & 34 & 50,7 \\
Sim & 33 & 49,3 \\
Total & 67 & 100,0 \\
\hline
\end{tabular}

Com quem pode contar se precisar de ajuda?

\begin{tabular}{lcc} 
Ninguém & 25 & 37,3 \\
Parceiros e parentes & 42 & 62,7 \\
Total & 67 & 100,0 \\
\hline
\end{tabular}

\section{Discussão}

A internação hospitalar por causas associadas ao uso de substâncias psicoativas é maior entre a população em vulnerabilidade socioeconômica e educacional, significando que políticas públicas que favoreçam o suporte social devem ser implementadas. Essas políticas surgiram com o planejamento terapêutico, integração terapêutica, interação interconsultas terapêuticas e emergências e 
IB na Europa, contribuindo para as discussões sobre a trajetória da saúde mental no Brasil(9).

No entanto, as comorbidades associadas ao uso de álcool e tabaco são doenças de interesse, principalmente, dos profissionais da atenção primária. Contudo, é um problema de saúde pública, dada a sua alta prevalência encontrada na sociedade nas últimas décadas ${ }^{(10)}$.

Nesse sentido, é de fundamental importância o diagnóstico precoce de doenças associadas ao uso de SPA nas unidades básicas de saúde, por meio das entrevistas realizadas por profissionais capacitados, que podem utilizar conhecimentos e técnicas de Intervenção Breve (IB) junto aos programas de saúde pública(11). Evidenciou-se, ademais, que 41,4\% desconheciam qualquer problema de saúde previamente, o que corrobora a necessidade da prática da IB para aprimorar a prevenção ao uso e abuso das substâncias psicoativas.

Contudo, é comum que os transtornos de uso de substância psicoativa e comorbidades sejam diagnosticados em instituições hospitalares, ao serem estes pacientes atendidos em situações de emergência por complicações clínicas. Desse modo, é importante a atuação de profissionais com conhecimento e capacitação para oferecer atendimento com qualidade assistencial em saúde mental, possibilitando a utilização do método para aplicação da IB ${ }^{(12)}$.

No mesmo objetivo de aprimorar a qualidade do cuidado prestado, a Organização Nacional de Acreditação Hospitalar (ONA) descreve que as instituições hospitalares acreditadas em nível 1 devem ter procedimentos para atender pacientes vítimas de agressão física, moral, psicológica, abandono e tentativa de suicídio, assim como prestar ao paciente e aos seus familiares, apoio quanto às questões socioeconômicas e a reintegração social. Infere-se, assim, a importância da atuação de uma equipe multiprofissional com capacitação em saúde mental para realizar o atendimento a esses indivíduos admitidos na rede hospitalar ${ }^{(13)}$.

Contudo, múltiplos fatores como o dimensionamento de pessoal, custos de atendimento médico especializado, podem interferir no atendimento multiprofissional ao indivíduo com comprometimento da saúde mental, seja atendimento intra-hospitalar ou encaminhamento para reabilitação.

O tempo médio de permanência em pessoas com quadro de abuso de substâncias psicoativas, têm muitas vezes sofrido aumento, quando associado às dificuldades socioeconômicas, apontando a dificuldade da alta hospitalar devido ao fator sociodemográfico, que tem como impacto diretamente a falta de renda. Tal fato demonstra também a necessidade de atenção do serviço social(14).

Neste contexto, múltiplos fatores podem contribuir para um tempo médio de permanência de internação, levando ao atraso na alta hospitalar, designadamente relacionado com os serviços sociais. Dada esta tendência, três tipos de causas podem interferir diretamente no atraso da alta hospitalar: causas organizacionais ou intrahospitalares como a fragilidade no dimensionamento de profissional especializado para o manejo das complicações do indivíduo quanto à saúde mental tendo como exemplo o manejo e controle dos sinais e sintomas da abstinência; causas individuais como a aceitação do tratamento proposto e este estar relacionado ao uso de SPA podendo o indivíduo estar na fase de pré-contemplação ou contemplação e causas da comunidade relacionadas a recursos existentes na comunidade para o atendimento ao indivíduo, onde nem sempre proporciona a continuidade e acompanhamento do atendimento proposto do manejo da saúde mental(15).

Quanto às causas organizacionais citadas anteriormente, considera-se de grande importância a atuação multiprofissional, sendo que há estudo realizado na Inglaterra com 179 hospitais, dos quais 168 gozavam de serviços de ligação em psiquiatria, sendo que $79 \%$ destes incluíam na equipe ao menos um psiquiatra e um enfermeiro especialista em saúde mental, equipe essa que prestava atendimento em todas as unidades do hospital. No entanto, o estudo não relata qual o percentual de atendimento dos indivíduos internados que necessitavam de atendimento da equipe. A principal causa de admissão neste estudo foi por automutilação (63\%) causada pelo abuso de álcool e de outras substâncias ${ }^{(16)}$. O processo de atendimento ao indivíduo por meio de uma equipe multiprofissional, além de promover melhor relações humanas, tem como objetivo realizar um tratamento individualizado, planejado e efetivo em todos os aspectos e necessidades biopsicossocial do indivíduo(13).

Por tempos, as interconsultas somente eram solicitadas entre os profissionais médicos, o que tornava ostensivas as poucas solicitações para a equipe de atuação em saúde mental. Neste novo âmbito hospitalar, onde se deu a valorização multiprofissional, outros profissionais passaram também a solicitar avaliações e interconsultas aos indivíduos elegíveis para tratamento em saúde mental, englobando os que fazem uso abusivo de substância psicoativa(17).

Não sendo diferente nesta pesquisa, há tempos a interconsulta era solicitada somente pelo profissional médico, com o passar dos anos e a implantação do processo de segurança do paciente as solicitações de interconsulta podem ser realizadas por qualquer profissional da área de saúde aos profissionais de psicologia, serviço social e enfermagem, para a solicitação do profissional psiquiatra ainda se faz necessário a solicitação de médico para médico, por questões de documento e faturamento da auditoria financeira. 
No presente estudo, foi observado que as interconsultas eram minimamente solicitadas ao profissional especializado em saúde mental, considerava-se que o indivíduo buscou ajuda hospitalar por questões de complicações clinicas ou traumas, assim o atendimento era realizado pelo profissional específico da complicação orgânica encontrada. As alterações psicológicas e/ou psiquiátricas que apresentava, seja pela abstinência ou pelo uso excessivo da SPA, eram tratadas pelo profissional médico responsável pela internação primária, e quando esta comprometia o tratamento clinico solicitava-se o atendimento específico e serviço social para programar a alta para continuidade após a alta hospitalar.

Em outro estudo, realizado em Lisboa, foi também avaliado o percentual das interconsultas aos profissionais especialistas em saúde mental, encontrando-se solicitações de interconsulta para 750 pacientes durante cinco anos. Foi identificado que $25 \%$ desses pedidos tiveram como motivo a depressão e $19 \%$ foram por agitação psicomotora, considerando perturbações mentais orgânicas agudas, sendo que $60 \%$ dos pacientes não tinham acompanhamento prévio em psiquiatria(18).

Todavia, interconsultas para o atendimento da saúde mental nem sempre são bem aceitas nos serviços gerais. Estudo realizado na Polônia sugeriu que apenas $16 \%$ dos psiquiatras têm interação clínica com os demais especialistas disponíveis no hospital geral(19).

Corroborando com as pesquisas já realizadas, este estudo também observou a fragilidade para alta hospitalar de forma efetiva para os casos em que o indivíduo necessitava de apoio do município para a continuidade do tratamento em saúde mental, uma vez que não abrange e não disponibiliza atendimento imediato após a alta, tendo assim o serviço social da instituição a dificuldade de otimizar a alta hospitalar.

Soma-se o fato de que há dificuldade para alta, e de o hospital também ter como fator a descontinuidade do cuidado, acarretando insuficiência de serviços e profissionais na rede de serviços psiquiátricos públicos para o encaminhamento dos pacientes ao serviço especializado(20). Na instituição pesquisada, foi observada uma fragilidade no processo de solicitação de interconsulta, uma vez que não foi evidenciada a disponibilidade do profissional médico psiquiatra em escala de plantão, assim como nos dados levantados via sistema apresentados nos resultados quanto à avaliação de especialista. O que podemos indagar que contribuiu para o aumento do tempo médio de permanecia nas UTIs e nos setores de internação.

Em estudo realizado com quatro grupos, 31 indivíduos, observou-se que a busca por ajuda se deu a partir da instalação da dependência química ou por esses indivíduos terem perdido algo significativo em sua vida, em que o usuário transpassou por internações hospitalares decorrentes e complicações ${ }^{(21)}$, assim como também é sabido que o uso de SPA está associado ao desenvolvimento de comorbidades e propensão a crimes violentos, levando a enfatizar a necessidade de atenção interdisciplinar ao usuário de SPA, uma vez que estão vulneráveis a problemas socioeconômicos, culturais e familiares ${ }^{(22)}$, tal como vem sendo debatido por gestores, pesquisadores e operadores de políticas sociais. No Brasil, tal debate pode ser exemplificado por uma revisão integrativa baseada na pergunta "Quais são os indicadores de vulnerabilidade relacionados à questão social apresentados em estudos científicos e como eles são construídos? ${ }^{(23)}$.

Em estudo com 29 casos, que estavam relacionados a eventos sentinelas, 93,3\% desses resultaram em internação hospitalar pelo uso crônico de bebida alcoólica. Tendo uma média de abuso do álcool de 20,8 anos, 55,2\% dessas famílias tinham comportamento aditivo ao uso de substâncias psicoativas, tendo casos com mais de 20 anos de abuso do álcool. Apenas 10,3\% dessas famílias não foram consideradas vulneráveis em questões sociais como desemprego, baixa escolaridade, uso diário de droga, o que permitiu concluir que o uso de SPA agravou as condições de vulnerabilidade das famílias ${ }^{(23)}$.

No âmbito da pesquisa qualitativa, foi feito o levantamento da percepção dos familiares quanto a mudanças no cotidiano, seja familiar e/ou social, após a inserção da dependência de drogas. Os relatos demonstraram que a convivência com pessoas dependentes de substâncias psicoativas pode desencadear vários tipos de sentimentos, emoções e expectativas para uma mudança por meio da fé, assim como fica evidente o sentimento de medo(24).

No contexto religioso e espiritual, orações, penitências e jejuns foram métodos utilizados por familiares como estratégias para buscar força em Deus, sendo as orações uma forma de aproximação com a força superior para alcançar forças para resistir às adversidades diárias ${ }^{(25)}$. A busca por orações e crenças religiosas tanto pelos pacientes quanto pelos familiares neste momento ficou evidente no decorrer da pesquisa, ambos em busca de conforto e "cura". Buscam na oração uma força interna para conseguir superar as dificuldades encontradas no momento.

As implicações do uso de substâncias psicoativas apontam principalmente para o uso de álcool no âmbito familiar, que pode influenciar o uso de drogas ilícitas precocemente, podendo ser na infância ou na adolescência, como encontrado nesta pesquisa, que 40,3\% dos indivíduos convivem com familiares que também fazem uso de SPA, o que vem contribuir para o estudo qualitativo realizado na região metropolitana da Baixada Santista-SP, com usuários de crack, identificou nos relatos que o uso de drogas ilícitas com início na adolescência 
ocorreu com o consentimento ou compartilhamento dos próprios pais. Destacando também o comportamento agressivo e violento dos pais, sendo o pai o maior agressor quando se encontrava sob efeito de drogas ${ }^{(26)}$.

Estudo apontou que entre diversos fatores do uso precoce de drogas destaca-se, na ótica do usuário de crack, a fragilidade dos vínculos familiares, com presença de violência doméstica na infância, conflitos, perdas afetivas, separações das figuras parentais e o uso de álcool ou outras drogas ilícitas entre os familiares de seu convívio. Em 2009, avaliou-se também a presença de violência infantil associada ao uso de SPA, identificando que muitos não consideraram a agressão como ato de violência, por ser uma prática comum e aceita culturalmente como uma prática educativa(26-27).

Podemos inferir, a partir dos estudos citados, que valores, questões culturais, composição familiar e convívio social interferem nas relações afetivas, sendo a violência doméstica o fator de maior relevância e impacto para influenciar precocemente o indivíduo ao uso de SPA, dificultando a harmonia familiar e social, o que reforça para este estudo que foi identificado que $44,8 \%$ dos indivíduos sofreram agressão física em algum momento da sua vida e destes $71 \%$ ainda quando criança menor que 10 anos, sendo que $52,2 \%$ do total pesquisado convivem no momento com outros familiares que também fazem uso de SPA.

Estabelecer uma relação de confiança e cuidado da saúde mental entre os familiares e os usuários de SPA é uma meta ainda a ser atingida pelos profissionais, uma vez que as necessidades de saúde ainda são vinculadas a saúde física, pois o que não se manifesta por meio de sinais e sintomas nem sempre é reconhecido como uma necessidade ${ }^{(28)}$. Exposição essa que nos mostra a falta de consciência para com as doenças que afetam o comportamento e saúde mental.

Este autor corrobora também que o convívio com dependentes de drogas ilícitas, como o crack, por exemplo, se dificulta ainda mais quando os danos na família são frequentes, como a venda dos próprios pertences e dos familiares em busca de renda para a manutenção do vício, levando inclusive a riscos decorrentes de furtos. A utilização da substância faz com que a cada dia os integrantes da família se afastem, pois não permite uma relação de reciprocidade, levando à perda dos vínculos(26-29).

A pesquisa qualitativa com familiares levantou relatos e percepções de que alguns fatos, como a existência de uma pessoa importante para o indivíduo, podem ser motivacionais para, ao menos, o dependente de SPA tentar se manter na abstinência. Houve também relatos de momentos de afeto positivo e diversão no convívio familiar que possibilitaram a recaída para o uso de drogas, assim como afetos negativos ${ }^{(24)}$. Este contexto verifica-se na pesquisa em questão, em que $40,3 \%$ dos pacientes têm como apoio familiares que também fazem uso de substancia psicoativas, e $47,8 \%$ de pessoas do seu convívio não fazem uso de nenhuma substância o que nos sugere que estes terão maior motivação para se manter na abstinência.

A busca pelo reconhecimento social de que o abuso de SPA deve ser considerado como doença e não como estado moral é inesgotável, assim como a busca para melhoria da qualidade no atendimento a esses indivíduos.

Este estudo identificou a exclusão social de usuários de SPA, que em algum momento estiveram expostos à situação de vulnerabilidade e possíveis situações de violência. Sendo assim, será importante e necessário restaurar o compromisso ético do Estado quanto aos princípios protetivos a essas pessoas, propondo evolução e adequação das estratégias de acolhimento ao indivíduo e capacitação especializada dos profissionais.

Corrobora com estudo qualitativo, realizado em uma unidade de saúde, que objetivou avaliar o método de ensino e aprendizado na formação profissional e nas capacitações nas instituições de saúde, concluindo que, por meio das metodologias ativas, o aprendizado dos colaboradores é mais efeito e tem resultado mais positivo quando as capacitações são realizadas continuadamente ${ }^{(30)}$.

\section{Conclusão}

A busca por ajuda e atendimento hospitalar nos exibe a fragilidade do corpo primeiramente, onde buscam da cura da dor. No entanto, nos deparamos com a necessidade do atendimento concomitantemente da saúde mental pelo uso da SPA, que necessita de uma equipe especializada ativa o que não foi encontrado neste cenário de pesquisa, devido à fragilidade na solicitação de interconsulta e numero de profissionais não compatíveis com a demanda.

Esta avaliação de profissionais especializados em saúde mental não é realizada na devida proporção, uma vez que pode haver interferência quanto à verba de custeio do SUS por não conseguir suprir financeiramente a prestação de serviços de profissionais especialistas em saúde mental, apesar de orientação da ONA, como requisito para gestão de qualidade e segurança, para que os indivíduos vítimas de agressão física, moral, psicológica, abandono e tentativa de suicídio recebam atendimento, dado que $44,8 \%$ dos indivíduos em uso de álcool apresentaram pontuação maior que 27 , necessitando, portanto, de encaminhamento ao serviço de atendimento especializado para reabilitação da saúde mental, custos estes que deverão ser detalhados posteriormente em outro estudo.

São necessárias novas pesquisas nesta temática, com o objetivo de avaliar o desfecho e suas complicações clínicas após a implementação de estratégias, como 
interconsulta e acompanhamento multiprofissional em saúde mental, de forma a minimizar a permanência e custos da internação hospitalar.

\section{Referências}

1. Amarante $P$, Torre EHG. Madness and cultural diversity: innovation and rupture in experiences of art and culture from Psychiatric Reform and the field of Mental Health in Brazil. Interface. 2017;21(63):763-74. doi: http://doi. org/10.1590/1807-57622016.0881

2. Nascimento LR. Reforma psiquiátrica brasileira [dissertação]. Goiânia: Universidade Federal de Goiás; 2018.

3. World Health Organization. Global status report on alcohol and health. Geneva: WHO; 2018.

4. Portaria no 3.088, de 23 de dezembro de 2011 (BR). Institui a Rede de Atenção Psicossocial para pessoas com sofrimento ou transtorno mental e com necessidades decorrentes do uso de crack, álcool e outras drogas, no âmbito do Sistema Único de Saúde (SUS). Diário Oficial da União. [internet]. 26 dez 2011. [Acesso 8 out 2019]. Disponível em: https://bit.ly/29zD847

5. Ministério da Saúde (BR). Direitos humanos e saúde mental. [Internet]. 2014 Nov 27 [Acesso 27 nov 2014]. Disponível em: https://bit.ly/2nrylOa

6. Pacheco AM, Silva RDMS, Ramos MC. Designing a health care work flow for alcohol and drug addiction. Rev Bras Pesqui Saúde. [Internet]. 2017 [cited 2019 Oct 8];19(1):21-7. Available from: http://periodicos.ufes.br/ RBPS/article/download/17712/12138

7. Henrique IFS, De Micheli D, Lacerda RB, Lacerda LA, Formigoni MLOS. Validação da versão Brasileira do teste de triagem do envolvimento com álcool, cigarro e outras substâncias (ASSIST). Rev Assoc Med Bras. 2004;50(2):199-20. doi: http://dx.doi.org/10.1590/ S0104-42302004000200039

8. Gorenstein C, Yuan-Pang W, Hungerbühler I, compiladores. Instrumentos de Avaliação em Saúde Mental. Porto Alegre: Artmed; 2016. 500 p.

9. Botega NJ. Prática psiquiátrica no hospital geral: interconsulta e emergência. 4.ed. Porto Alegre: Artmed; 2017.

10. World Health Organization. Substance Abuse Department. Global status report: alcohol policy. Geneva: WHO; 2019. [Acesso 1 out 2020]. Disponível em: https:// www.who.int/substance_abuse/activities/gsrah/en/ 11. Abreu AMM, Jomar RT, Taets GGC, Souza MHN, Fernandes DB. Screening and brief intervention for the use of alcohol and other drugs. Rev Bras Enferm. 2018;71(Suppl 5):2258-63. doi: http://doi. org/10.1590/0034-7167-2017-0444

12. Jaworowski S, Raveh-Brawer D, Gropp C, Haber PS, Golmard JL, Mergui J. Alcohol related harm: knowledge assessment of medical and nursing staff in a general hospital. Isr J Psychiatry. [Internet]. 2018 [cited 2019 Oct 8];55(2):32-6. Available from: https://www.ncbi. nlm.nih.gov/pubmed/30351278

13. Organização Nacional de Acreditação (BR). Manual das Organizações Prestadoras de Serviço em Saúde. São Paulo: Organização Nacional de Acreditação; 2018. 152 p. 14. Baeza FL, Rocha NS, Fleck MP. Predictors of length of stay in an acute psychiatric inpatient facility in a general hospital: a prospective study. Rev Bras Psiquiatr. 2018;40(1):89-96. doi: http://doi. org/10.1590/1516-4446-2016-2155

15. Modas DAS, Nunes EMGT, Charepe, ZB. Causas de atraso na alta hospitalar no cliente adulto: scoping review. Rev Gaúcha Enferm. 2019;40:e20180130. doi: http://doi. org/10.1590/1983-1447.2019.20180130

16. Walker A, Barrett JR, Lee W, West RM, Guthrie E, Trigwell $P$, et al. Organisation and delivery of liaison psychiatry services in general hospitals in England: results of a national survey. BMJ Open. 2018;8:e023091. doi: http://doi.org/10.1136/bmjopen-2018-023091

17. Paula BHB. Estudo das relações interprofissionais no hospital geral: contribuições da saúde mental para uma clínica do sujeito [dissertação]. Campinas: Universidade Estadual de Campinas; 2016.

18. Silva IC, Lopes J. 5 anos de psiquiatria de ligação no Hospital Vila Franca de Xira. Rev Psiquiatr Consil Ligac. [Internet]. 2019 [Acesso 8 out 2019];26(1):1-4. Available from: https://bit.ly/2osdomR

19. Chojnowski J, Załuska M. Psychiatric wards in general hospitals: the opinions of psychiatrists employed there. Psychiatr Pol. 2016;50(2):431-43. doi: http://doi. org/10.12740/PP/35378

20. Andrade ACM, Otani MAP, Higa EFR, Marin MJS, Caputo VG. Multiprofessional care in a psychiatric unit of general hospital. Rev Psicol Diversidade Saúde. 2018;7(1):60-71. doi: http://doi.org/10.17267/2317-3394rpds.v7i1.1846 21. Azevedo CF. Manejo do uso abusivo de álcool e outras drogas na perspectiva da entrevista motivacional [dissertação]. Goiânia: Universidade Federal de Goiás; 2015.

22. Claro HG, Oliveira MAF, Titus JC, Fernandes IFAL, Pinho PH, Tarifa RR. Drug use, mental health and problems related to crime and violence: cross-sectional study. Rev. Latino-Am. Enfermagem. 2015;23(6):1173-80. doi: http://doi.org/10.1590/0104-1169.0478.2663

23. Schumann LA, Moura LB. Vulnerability synthetic indices: a literature integrative review. Ciênc Saúde Coletiva. 2015;20(7):2105-20. doi: http://doi. org/10.1590/1413-81232015207.10742014

24. Rodrigues TFCS. Feelings of families regarding drug dependence: in the light of comprehensive sociology. Rev Bras Enferm. 2018;71(Suppl 5):2272-9. doi: http://doi. org/10.1590/0034-7167-2018-0150 
25. Claus MIS, Zerbetto SR, Gonçalves AMS, Galon T, Andrade LGZ, Oliveira FC. The family strengths in the context of psychoactive substance dependence. Esc Anna Nery. 2018;22(4):e20180180. doi: http://doi. org/10.1590/2177-9465-EAN-2018-0180

26. Barros NA, Tucci AM. Perceptions of crack users on their family relationships in childhood and adolescence. Psicol Teoria Pesqui. 2018;34:e34419. doi: http://doi. org/10.1590/0102.3772e34418

27. Dunlap E, Golub A, Johnson BD, Benoit E. Normalization of violence: experiences of childhood abuse by inner-city crack users. J Ethn Subst Abuse. 2009;8(1):15-34. doi: http://doi.org/10.1080/15332640802683359

28. Lima DWC, Leite ACQB, Vieira AN, Leite AR, Luis MAV, Azevedo LDS, et al. Necessidades de saúde de familiares de usuários de substâncias psicoativas. Rev Eletron Enferm. [Internet]. 2018 [cited 2019 Oct 8];20:v20a12. doi: http://doi.org/10.5216/ree.v20.47410

29. Siqueira DF. Familiar do usuário de substâncias psicoativas: revisão de literatura. Universidade Regional Integrada do Alto Uruguai e das Missões - Campus Santiago. 2018. [Acesso 1 out 2020]. Disponível m: http://www.urisantiago.br/multicienciaonline/adm/upload/ v3/n5/02fe22060bbb9f546f81229f7228e6f9.pdf

30. Fernandes MA, Soares NSA, Ribeiro ÍAP, Sousa CCMS, Ribeiro HKP. Active methodologies as a tool for training in mental health. Rev Enferm UFPE on line. 2018;12(12):3172-80. doi: http://doi.org/10.5205/19818963-v12i12a237762p3172-3180-2018

\section{Contribuição dos autores}

Concepção e planejamento do estudo: Jaqueline Fátima de Souza. Obtenção dos dados: Jessica Andrade Tiziani. Revisão crítica do manuscrito: Marcos Hirata Soares.

Todos os autores aprovaram a versão final do texto. Conflito de interesse: os autores declararam que não há conflito de interesse. 